\title{
Effect of submicron deformations on the transmission of all-solid photonic bandgap fibre
}

\author{
Andrey I. Konyukhov*, Elena A. Romanova \\ Physics Department, Saratov State University, Saratov, Russia \\ *e-mail: kai@optics.sgu.ru \\ Trevor M. Benson, Giorgos S. Athanasiou \\ George Green Institute for Electromagnetics Research \\ Faculty of Engineering, University of Nottingham, Nottingham, UK \\ Joris Lousteau \\ Optoelectronics Research Centre \\ University of Southampton, Southampton, UK \\ Gerardo Scarpignato, Diego Pugliese, Daniel Milanese \\ Politecnico di Torino, Turin, Italy
}

\begin{abstract}
Waveguide losses in all-solid photonic bandgap fibre are studied numerically using both vectorial mode solver and vectorial beam propagation methods. Confinement loss, including material losses, is comprehensively evaluated for defect modes of hexagonal lattice photonic bandgap fibre. The excitation of the index-guiding modes at the bandgap edges leads to a narrowing of the transmission bands. Submicron deformations of the transverse structure of the fibre lead to a significant reduction in the width of the transmission bands, yet the minimum value of the losses within these bandgaps remains practically unchanged. Longitudinal variation of the fibre profile increases the effective losses by up to tens of $\mathrm{dB} / \mathrm{m}$.
\end{abstract}

Keywords modelling, photonic bandgap fibre, optical fibre losses.

\section{Introduction}

Dhotonic bandgap fibre (PBF) guides light by exploiting photonic bandgap effects. Solid-core PBF typically has a low refractive index core surrounded by a periodically-arranged cladding composed of high-index rods. The core region corresponds to a defect in the centre of the periodic structure (Argyros et al., 2005)]. The waveguiding properties of PBF give great potential for the use of PBF in, for example, nonlinear fibre optics (Vanvincq et al., 2012), optical sensing (de Oliveira et al., 2013) and wavelength filtering (Yan et al., 2010). Solid-core PBF is much less sensitive to bending than a step-index singlemode fibre (Ren et al., 2013). Effectively-single-mode all-solid photonic bandgap fibre can be designed with large effective mode area and low bending loss for high-power laser applications (Saitoh et al., 2014). The internal structure of all-solid PBFs is protected from the effects of the atmosphere, whereas air-glass micro-structured optical fibres can show optical and structural aging when stored under ambient atmospheric conditions (Toupin et al., 2014; Mouawad et al., 2014).

Fibre losses and their spectral dependency are critical in the functioning of the PBF. A special design of high-index rods with an index depressed layer allows losses of $2 \mathrm{~dB} / \mathrm{km}$ to be achieved (Ren et al., 2013). Transmission losses as low as $0.41 \mathrm{~dB} / \mathrm{km}$ have been reported for fused-silica all-solid PBF (Saitoh et al., 2014). Non-silica glass PBFs typically have higher transmission losses than fused-silica ones. In tellurite-filled silica photonic crystal fibre a minimum loss of $1.6 \mathrm{~dB} / \mathrm{cm}$ was achieved (Schmidt et al., 2009). Chalcogenide glass PBFs with interstitial air holes are described by (Brilland et al., 2008). For GeSbS and AsSebased PCFs the best transmission losses were, respectively, $3 \mathrm{~dB} / \mathrm{m}$ and $5 \mathrm{~dB} / \mathrm{m}$ at $1.55 \mu \mathrm{m}$. For a TeAsSe PCF the lowest transmission loss was $6 \mathrm{~dB} / \mathrm{m}$, at a wavelength of $9.3 \mu \mathrm{m}$. With collapsed interstitials holes the transmission of all the fibres was greater than $20 \mathrm{~dB} / \mathrm{m}$. All-solid tellurite-glass photonic crystal fibre had experimentally measured transmission losses of around $50 \mathrm{~dB} / \mathrm{m}$ (Lousteau et al., 2012). For chalcogenide glass PBF, (Caillaud et al., 2014) measured losses in the 20-50 dB/m range at 
a wavelength of $3.39 \mu \mathrm{m}$. High purity tellurite glasses, with $10^{-3} \mathrm{~dB} / \mathrm{km}$ losses (Hill and Jha, 2007), and chalcogenide glass fibres, with losses as low as $12 \mathrm{~dB} / \mathrm{km}$ (Snopatin et al., 2009), can be produced. However, the experimental losses in non-silica PBFs are still significantly higher than the intrinsic material losses (Schmidt et al., 2009; Ren et al., 2013; Saitoh et al., 2014; Lousteau et al., 2012; Caillaud et al., 2014). The increased loss in the high-transmission windows may be related to the scattering at the interfaces due to the formation of crystallites by glass devitrification (Schmidt et al., 2009) or to the formation of air bubbles in the fibre preform (Brilland et al., 2008). Another origin of increased losses is the deformation of the PBF structure. During drawdown of glass-air micro-structured optical fibres, a range of hole deformation patterns can occur (Xue $e t$ al., 2006), including hole collapse, hole expansion, hole enlargement and hole shape changes. All-solid PBFs are more robust towards structural deformations; however, unwanted structure changes can also be found (Brilland et al., 2008; Lousteau et al., 2012; Caillaud et al., 2014). Transversal defects due to the fabrication process enhance field tunnelling through the PBG into the outer cladding. Structural distortions in air-core photonic bandgap fibres have significant impact on fibre properties such as bandwidth and tunnelling loss. Numerical results (Li et al., 2007) show that the bandwidth of the real fibre is reduced by a factor of two, and that the tunnelling is increased by a factor of almost $10^{4}$ when compared to an ideal cross-section. The impact of transversal defects on the minimum confinement loss value in the case of a solid-core photonic-bandgap fibre is described by (Pureur et al., 2007). A deviation of only 5\% in the diameter, the refractive-index contrast, or the position of the high-index inclusions could double the minimal value of losses within the third-order photonic bandgap.

The experimental losses are still significantly higher than the theoretical ones, even if the intrinsic material losses are taken into account in the simulations (Ren et al., 2013; Caillaud et al., 2014]. To reveal the possible origins of large transmission losses, in this paper we consider the influence of both the transverse deformation of PBF structure and the longitudinal variation of the fibre geometry. We study the effects of structural distortions of the real fibre on PBF transmission and compare it to a similar ideal PBF without distortions. For the calculation of the confinement losse of selected mode, the plane-wave expansion method (Sakoda 2001; Melnikov et al., 2005) was applied. To consider the excitation of few modes and the effect of the variation of the fibre profile along its length, the Fourier-based beam propagation method (Wangüemert-Pérez et al., 2004) was used. For the PBF fibre considered, the deformation of the transverse structure leads mainly to a narrowing of transmission bands. To reveal the possible source of large experimental losses observed in a real all-solid PBF (Lousteau et al., 2012), a submicron longitudinal variation of the fibre geometry was studied.

\section{Modes of Photonic Bandgap Fibre}

Fig. 1a shows the transverse structure of the experimental fibre considered (Lousteau et al., 2012). The fibre consists of highrefractive index micro-sized rods packed into a hexagonal lattice. The refractive indices of the rods and the host glass are shown in Fig. 1b. The aimed-for hexagonal lattice was slightly deformed during the drawing process. The supercell (Fig. 1a) chosen for the calculation of the PBF modes is characterized by the angle $\theta=64^{\circ}$. The distance between high-index rods $\Lambda$ varies in the range $8.6 \mu \mathrm{m} \pm 0.3 \mu \mathrm{m}$. The diameters of the rods are $2.25 \mu \mathrm{m} \pm 0.1 \mu \mathrm{m}$. The cross-section of the rods is elliptical. The ratio of the length of the major ellipse axis to the minor one varies from 1.06 to 1.31 .

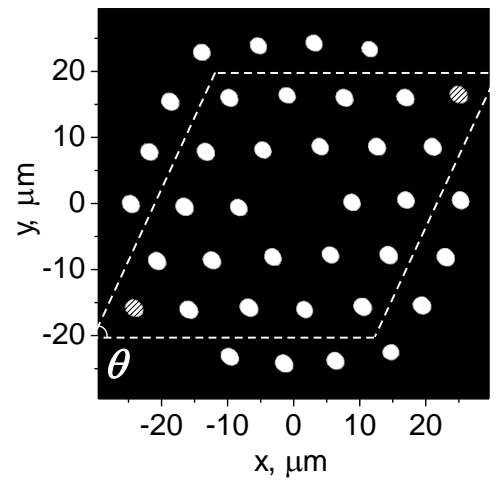

(a)

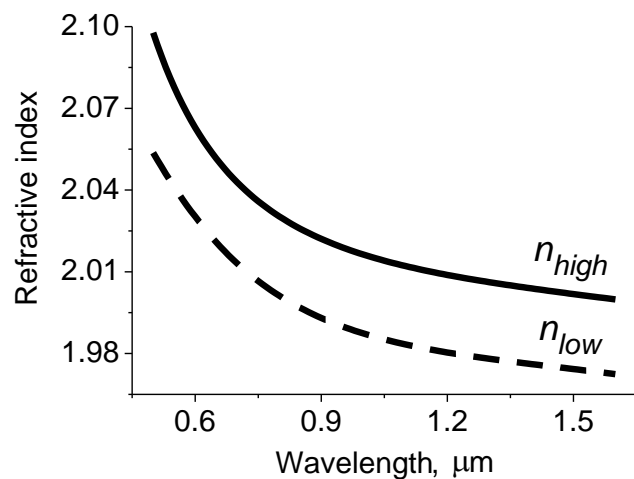

(b)

Fig. 1 Structure of the real PBF. a) Refractive index profile. White corresponds to the high refractive index rods with $n_{\text {high }}$, black to the area with refractive index $n_{\text {low }}$. The area surrounded by the white dashed line is the supercell on which the numerical calculation of PBF modes was carried out. The angle of the diamond-shaped supercell is $\theta$. The supercell is completed by two high refractive index rods (dashed shading). (b) Wavelength dependency of the refractive indices of the rods, $n_{\text {high }}$, (solid curve) and the host glass, $n_{\text {low }}$, (dashed curve).

To study the effect of the structure deformation on the transmission spectrum of the fibre, both the experimentally realised PBF structure (Fig. 1a) and the idealized hexagonal lattice photonic bandgap fibre structure are considered. The hexagonal lattice 
is composed of circular dielectric rods. The diameters of the rods and the lattice period correspond to the average values of the real PBF structure (Fig.1). The supercell for the hexagonal lattice has $\theta=60^{\circ}$.

\subsection{PBF effective index diagram}

The modes of the PBF should satisfy the eigenvalue equation for the magnetic field vector $\boldsymbol{H}(\boldsymbol{r}, \omega)$ (Sakoda, 2001, Chapter 2):

$$
\mathcal{L}_{H} \boldsymbol{H}(\boldsymbol{r}, \omega)=(\omega / c)^{2} \boldsymbol{H}(\boldsymbol{r}, \omega)
$$

where $\mathcal{L}_{H}=\nabla \times\left(n^{-2}(x, y, \omega) \cdot[\nabla \times \boldsymbol{H}(\boldsymbol{r}, \omega)]\right), \omega$ is the angular frequency, $\boldsymbol{r}=(x, y, z)$ are the Cartesian coordinates, $c$ is the vacuum light velocity, $n(x, y, \omega)$ is the refractive index. For real refractive index $n_{r}=n(x, y, \omega)$ the operator $\mathcal{L}_{H}$ is Hermitian (Sakoda, 2001, Chapter 2) that reduces computational expenses sufficiently. However with the frequency-dependent refractive index $n_{r}(x, y, \omega)$ the eigenproblem (1) is nonlinear. Because the frequency $\omega$, which is necessary to calculate $n_{r}(\omega)$, is unknown. To overcome this difficulty, a scaling technique for wavenumber $(\omega / c)$ was used (Melnikov et al., 2005).

A plane-wave expansion method (Sakoda, 2001; Melnikov et al., 2005) was applied to the diamond-shaped supercell. The supercell for the real PBF structure has $\theta=64^{\circ}$ and $d=44.086 \mu \mathrm{m}$, where $\theta$ is the diamond's angle and $d$ is the length of the diamond side, as shown in Fig. 1a. To avoid the appearance of interstitial guiding cores, the supercell was completed by two artificial rods (dashed in Fig. 1a). The spatial Fourier coefficients for $n_{r}^{-2}(x, y)$ in (1) are calculated numerically using the bitmap image of the PBF end-face (Fig. 1a). The supercell for the idealized hexagonal lattice PBF has $\theta=60^{\circ}$ and $d=5 \Lambda=$ $43 \mu \mathrm{m}$. The spatial Fourier coefficients for dielectric rods with circular cross-section are calculated analytically. Equation (1) was solved for the eigenvalues $(\omega / c)$ at given value of longitudinal propagation constant $\beta_{0}$. The effective refractive index of the PBF mode is $n_{\text {eff }}=\beta_{0}(\omega / c)^{-1}=\beta_{0}(2 \pi / \lambda)^{-1}$.

The effective refractive index diagram for the real PBF structure (Fig. 1a) is shown in Fig. 2. Fig. 2 shows that the PBF has three bandgaps for $0.5 \mu \mathrm{m}<\lambda<1.6 \mu \mathrm{m}$. The first bandgap can be found at $\lambda>1.2 \mu \mathrm{m}$, the second one is localized at $0.75 \mu \mathrm{m}<\lambda<1.0 \mu \mathrm{m}$ and the third bandgap is localized at around $\lambda=0.6 \mu \mathrm{m}$.

In all-solid PBFs with high-index rods arranged in a low-index background the photonic band gaps arise from the coupling and broadening of the modes supported by the high-index rods (Lægsgaard, 2004). Given a band gap, introducing a defect in the crystal can produce localized states or so-called "defect modes" (Sakoda, 2001, Chapter 6). The considered fibre (Fig.1a) supports the propagation of two fundamental defect modes with orthogonal polarization. As an example, the transverse intensity distribution of the defect mode at the wavelength $\lambda=0.59 \mu \mathrm{m}$ is shown in Fig. 3a; the transverse intensity distribution for the fundamental defect mode shown in Fig. 3a is typical of that found within all the three bandgaps.

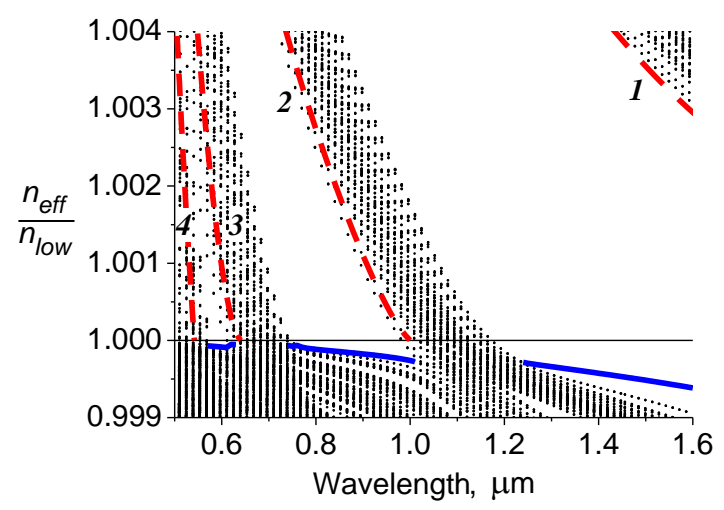

Fig. 2 Dispersion diagram for the PBF. The black dots are plotted for the real PBF structure (shown in Fig. 1a). Groups of index-guiding modes are indexed by the numbers $1,2,3$ and 4 . The dashed curves (red online) show the effective refractive index of the modes of a single stepindex circular fibre. The solid curves (blue online) show the effective refractive index of the fundamental defect mode. 

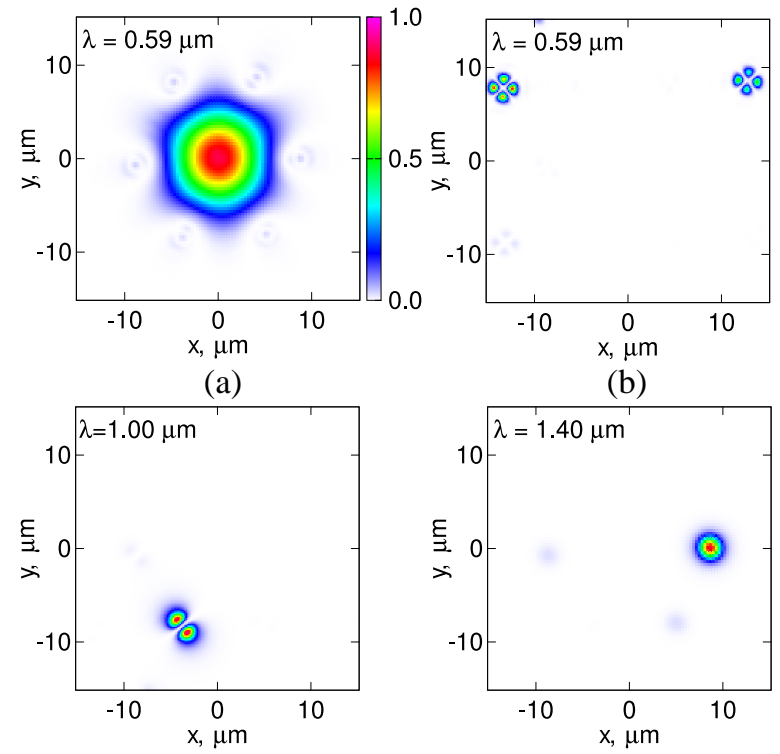

(c)

(d)

Fig. 3 Transverse intensity distribution for four modes of the PBF at different wavelengths $\lambda$. (a) Defect mode with $n_{\text {eff }} / n_{\text {low }}=0.999918$; (b) index guiding modes with $n_{\text {eff }} / n_{\text {low }}=1.00354$; (c) $n_{\text {eff }} / n_{\text {low }}=1.00094$; (d) $n_{\text {eff }} / n_{\text {low }}=1.00475$. The color bar in the Fig. 3a shows the intensity color mapping.

Defect modes have $n_{\text {eff }} / n_{\text {low }}<1$. Total internal reflection modes on the other hand have $n_{\text {eff }} / n_{\text {low }}>1$ (see Fig. $3 \mathrm{~b}$, c, d, for example). These modes are guided mostly within the high index rods, which act as step-index fibres. In Fig. 2 at least four groups of total internal reflection modes can be found. These groups can be described in terms of the modes of a step-index circular fibre. The dashed curves in Fig. 2 show the effective refractive index for the fundamental $H E_{11}$ mode $(1)$, the $T E_{01}$ mode (2), the $E H_{11}$ mode (3) and the $H E_{31}$ mode (4) of a single step-index circular fibre of core diameter $2.25 \mu \mathrm{m}$; the refractive index of the core is $n_{\text {high }}$ and the refractive index of the cladding is $n_{\text {low }}$.

Fig. 2 reveals that the wavelength $\lambda=1.2$ (Fig. 2) corresponds to the cut-off frequency of a high-order index guiding mode. Over the wavelength range $0.5 \mu \mathrm{m}<\lambda<1.2 \mu \mathrm{m}$ the coexistence of high-order index guiding modes and the defect mode guided by the central low index area (defect) is possible. Both the defect mode (Fig. 3a) and the high-order index guiding mode (Fig. 3b) can be excited at the same wavelength. For $\lambda>1.2 \mu \mathrm{m}$ each of the high index rods of the PBF supports the propagation of only a fundamental index guiding mode; a transverse intensity distribution that is typical of that for such a fundamental index guiding mode is shown in Fig. 3d.

The cross-section of the high index rods in the experimental PBF is not circular (Fig. 1a). Additionally, the shape of the high index rods is slightly variable from rod to rod. This leads to the removal of the degeneracy of the index guiding modes. Modes having similar symmetries in their transverse field distribution are grouped within a defined wavelength range in Fig. 2. The symmetry properties of the index-guiding modes can be described using the notation for the modes of a step-index circular fibre. We compare the dispersion characteristics of the PBF index-guiding modes with those of a circular step-index fibre whose diameter is equal to the average value of the diameter of the high refractive index rods in the PBF. The refractive index of the fibre core and cladding are $n_{\text {high }}$ and $n_{\text {low }}$, respectively (see Fig. 1b). Dashed curves in Fig. 2 show the dispersion properties of the fibre. The group of modes labelled as "1" in Fig. 2 corresponds to $H E_{11}$ modes. The second group (labelled as "2") belongs to $T E_{01}, T M_{01}$ and $H E_{21}$ modes. The third group corresponds to the $E H_{11}$ and $H E_{12}$ modes of the step-index fibre. The fourth group is formed by $\mathrm{EH}_{21}$ and $H E_{31}$ modes.

The dispersion diagram of a bandgap fibre having an idealized hexagonal lattice is shown in Fig. 4. As all the rods are identical, all the index guiding modes $\left(n_{\text {eff }} / n_{\text {low }}>1\right)$ are grouped with definite values that correspond to the dispersion properties of a single step-index circular fibre (Fig. 2, dashed curves). The cut-off wavelengths $\left(n_{\text {eff }} / n_{\text {low }}=0\right)$ of the index guiding modes are near to the "cut-off" of the defect mode (Fig. 4, solid curve). The wavelength ranges which correspond to the formation of the fundamental defect mode are very broad (Fig. 4) when compared to those for the real PBF (Fig. 2). The 
deformation of the fibre structure is observed to lead to a removal of the degeneracy of index guiding modes $\left(n_{\text {eff }} / n_{\text {low }}>1\right)$ and to the excitation of many space-filling modes $\left(n_{\text {eff }} / n_{\text {low }}<1\right)$ (Fig. 2$)$.

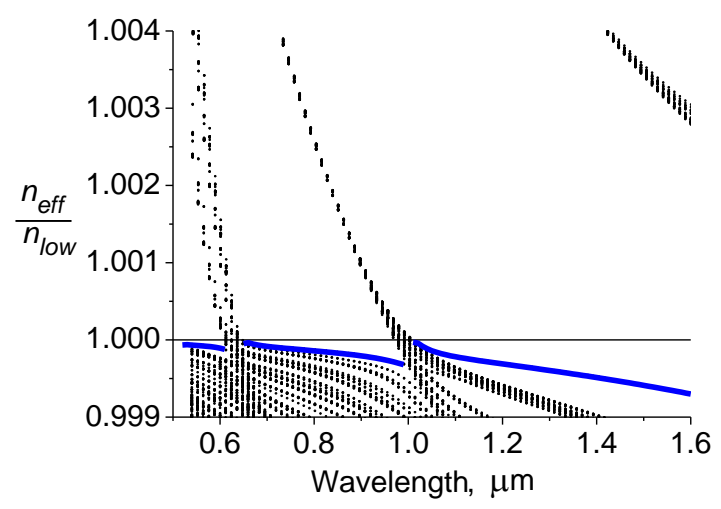

Fig. 4 Dispersion diagram of the PBF having an ideal hexagonal lattice $\left(\theta=60^{\circ}\right)$. Solid curves (blue online) show the effective refractive index of the fundamental defect mode.

\subsection{Confinement loss for fundamental defect mode}

The plane-wave method used above is useful for plotting effective index diagrams and for finding bandgap edges. To find the defect mode at a selected wavelength $\lambda$, the eigenvalue equation (1) can be written in another form. After the substitution of $\boldsymbol{H}(x, y, z)=\left(\mathbf{h}_{t}+\hat{\mathbf{z}} h_{z}\right) \exp \left(i \beta_{0} z\right)$ into (1) we have:

$$
\left(\nabla_{t}^{2}+k^{2} n^{2}\right) \mathbf{h}_{t}-\left(\nabla_{t} \times \mathbf{h}_{t}\right) \times\left(\nabla_{t} \ln n_{r}^{2}\right)=\beta_{0}^{2} \mathbf{h}_{t}
$$

Equation (2) allows one to find the eigenvalue $\beta_{0}^{2}$ and the eigenvector $\mathbf{h}_{t}=\left(h_{x}, h_{y}\right)$ at the given wavenumber $k=2 \pi \lambda^{-1}$. The magnetic field vector and the dielectric permittivity $\varepsilon=n_{r}^{2}$ are expanded in a $2 \mathrm{D}$ Fourier series. Using an iterative solver (Lehoucq et al, 1997), the solutions for selected modes can be found. The power loss coefficient $\gamma$ for the given mode can be calculated as follows (Snyder et al, 1983, section 18-25; Guobin et al., 2004):

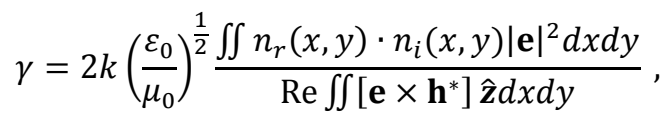

where $\varepsilon_{0}$ and $\mu_{0}$ are the vacuum permittivity and the vacuum permeability, respectively, and $\mathbf{e}$ and $\mathbf{h}$ are the electric and magnetic modal fields of the non-absorbing $\operatorname{PBF}\left(n_{i}=0\right)$. In evaluating (3), the imaginary part of the refractive index is taken as $n_{i}(x, y)=\alpha(x, y) \lambda(4 \pi)^{-1}$, where $\alpha$ is the material power losses coefficient and it is assumed that:

$$
\alpha(x, y)=\left\{\begin{array}{c}
2.3 \times 10^{-7} \mathrm{~m}^{-1}, r \leq 3 \Lambda \\
80 \mathrm{~m}^{-1}, r>3 \Lambda
\end{array}\right.
$$

where $r=\sqrt{x^{2}+y^{2}}$ and $\Lambda=8.6 \mu \mathrm{m}$. The absorption coefficient taken in the central area, $\alpha=2.3 \times 10^{-7} \mathrm{~m}^{-1}$, corresponds to a glass loss of $0.001 \mathrm{~dB} / \mathrm{km}$. Such a value can be attained in tellurite glasses in the mid-infrared wavelength range (Hill and Jha, 2007).

Equation (3) was derived using perturbation approach. For the correct application of the eq. (3) the imaginary part of the refractive index should be much less than the real one. For the chosen parameters (4) the value $n_{i}$ is less than $10^{-5}$ and $n_{r}$ does not exceed 2.1 (Fig.1b). So the condition $n_{i} \ll n_{r}$ is satisfied. The normalized effective refractive index of defect mode is $0.999<n_{\text {eff }} n_{\text {low }}^{-1}<1$ (Fig.2, Fig.4). Correspondingly we have $n_{i} \ll n_{\text {eff }}$. The supercell approximation becomes inaccurate for leaky modes, which fields exponentially increase with distance from the waveguide axis (Manenkov, 2005). Numerical modelling of leaky mode becomes possible only with adequate boundary conditions (Eliseev et al, 2005). Within photonic bandgaps defect mode is well localized (Fig.3a) and eq. 3 gives correct results (Guobin et al., 2004). At bandgap edges defect mode reaches cutoff. Since the supercell approximation becomes inaccurate if the guided mode occupies a sizeable fraction of the supercell, results are only reported for mode radius smaller than $1.74 \Lambda=15 \mu \mathrm{m}$. The mode radius is calculated using a fit of 
the transverse distribution of the $z$-component of time-averaged Poynting vector to a Gaussian function $\exp \left(-2 r^{2} / w^{2}\right)$. Fig.5 shows the mode radius $w$ both for the actual PBF structure and for the ideal hexagonal lattice PBF. In the wavelength range considered there are three bandgaps. Using the mode radius $w(\lambda)$, the wavelength ranges for the excitation of the fundamental defect modes can be easily found; they are summarised in Table 1 . The wavelength ranges shown in Table 1 were determined at the level $w=15 \mu \mathrm{m}$.

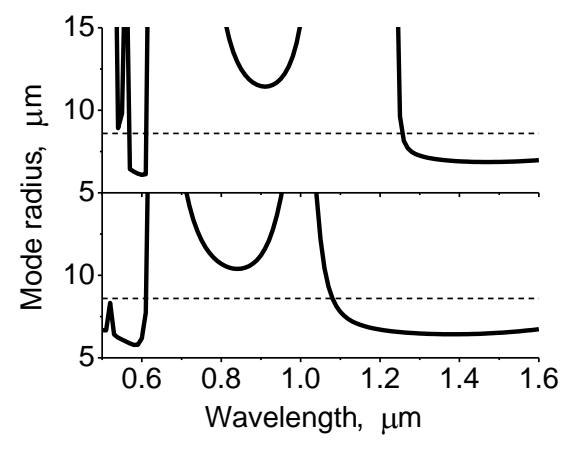

Fig. 5 PBF fundamental mode radius. The top panel is plotted for the actual PBF structure (Fig. 1a, $\theta=64^{\circ}$ ). The bottom panel is for the ideal hexagonal lattice $\operatorname{PBF}\left(\theta=60^{\circ}\right)$. The dashed line shows the structure period $\Lambda=8.6 \mu \mathrm{m}$.

At the first and at the third bandgaps the mode radius does not exceed the structure period $\Lambda$. The fundamental mode is thus well-confined to the centre of the PBF structure. Fig. 5 shows that at the second bandgap the mode radius exceeds the structure period. So the mode field overlaps to the high refractive index rods adjacent to the central defect of the PBF structure. In the presence of local scattering or bends an enhancement of the coupling between the fundamental defect mode and the indexguiding modes can therefore be expected.

The transmittance of $1 \mathrm{~m}$ long PBF was calculated based on Eqn. (3) and is shown in Fig. 6. There are three transmission bands that are in accordance with Table 1 . The wavelength ranges shown in the Table 1 correspond to the half-maximum transmittance for the given transmission band. The bandgaps numbers 1,2 and 3 in the Fig. 6 are shown. The local narrow peaks in the Fig. 6 (top panel) are due to the excitation of the index-guiding modes. The fundamental defect mode is absent at these wavelengths. Fig. 6 indicates that the transmittance in the second bandgap is lower than that in the first and third bandgaps. In general, the even-numbered bandgaps tend to suffer from high loss (Saitoh et al., 2014; Birks et al., 2006). The peak transmittance for the first and the third bands exceeds 0.99 , this corresponds to a loss of $0.04 \mathrm{~dB} / \mathrm{m}$. The transmission peak of the second band is 0.6 (corresponding to $2.1 \mathrm{~dB} / \mathrm{m}$ ). An increase of the power losses in the second passband was reported in (Lousteau et al., 2012) and this effect can now be associated with the mode expansion in the transverse cross-section shown in Fig. 5.

Table 1 Wavelength ranges for the fundamental defect mode.

\begin{tabular}{ccc}
\hline \hline Bandgap No. & Actual PBF & Hexagonal PBF \\
1 & $\lambda>1.23 \mu \mathrm{m}$ & $\lambda>1.04 \mu \mathrm{m}$ \\
2 & $0.8 \mu \mathrm{m}<\lambda<1.0 \mu \mathrm{m}$ & $0.71 \mu \mathrm{m}<\lambda<0.95 \mu \mathrm{m}$ \\
3 & $0.57 \mu \mathrm{m}<\lambda<0.61 \mu \mathrm{m}$ & $0.50 \mu \mathrm{m}<\lambda<0.61 \mu \mathrm{m}$ \\
\hline \hline
\end{tabular}

The transmittance of the PBF can be qualitatively described using exponential decay function $P(z) / P(0)=\exp (-\gamma z)$, which gives the relationship between initial power $P(0)$ and beam power $P(z)$ at the given propagation distance $z$ (Fig. 6). Fig. 6 shows that the deformation of the hexagonal structure and the submicron variation of the cross-section of high refractive index rods found in the experimental structure lead to a significant narrowing of the transmission bands. However, the peak value of the transmittance at the selected bandgap is practically the same both for the distorted PBF structure and the idealized hexagonal lattice PBF. 


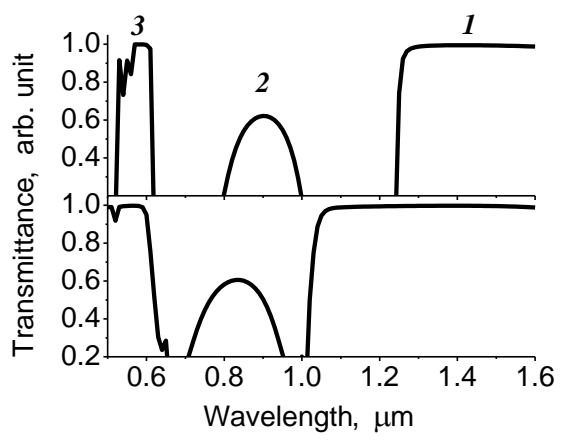

Fig. 6 Transmittance of the PBF calculated as $\exp (-\gamma \cdot z)$, where $z=1 \mathrm{~m}$. The top panel is for the real PBF structure (Fig. 1a, $\left.\theta=64^{\circ}\right)$. The bottom panel is for an ideal hexagonal lattice PBF $\left(\theta=60^{\circ}\right)$. The labels $1,2,3$ show the numbers of the corresponding bandgaps.

\section{Vector Beam Propagation Method}

In order to consider the effect of the longitudinal variation of the PBF structure on the fibre loss and to evaluate the impact of satellite index-guiding modes on the transmittance of the PBF, the vector beam propagation method (BPM) was employed.

\subsection{BPM equations}

The slowly varying amplitude of the magnetic field vector is considered, such that:

$$
\boldsymbol{H}(x, y, z)=\left(\widehat{\boldsymbol{x}} H_{x}+\widehat{\boldsymbol{y}} H_{y}+\hat{\mathbf{z}} H_{z}\right) \exp (i \beta z),
$$

where $\beta=2 \pi \lambda^{-1} n_{\text {low }}$ is the reference propagation constant. After the substitution of Eqn. (5) into Eqn. (1), two coupled equations can be obtained for $H_{x}$ and $H_{y}$. A major approximation that greatly simplifies the mathematical formulation is to assume that the terms $\left(\partial \ln n^{2} / \partial z\right)\left(\partial H_{z} / \partial x\right)$ and $\left(\partial \ln n^{2} / \partial z\right)\left(\partial H_{z} / \partial y\right)$ are neglected. Under these conditions, the components $H_{x}$ and $H_{y}$ satisfy:

$$
\begin{aligned}
& \left(1+\frac{i}{2 \beta} \frac{\partial \ln n^{2}}{\partial z}-\frac{i}{2 \beta} \frac{\partial}{\partial z}\right) \frac{\partial H_{x}}{\partial z}=\frac{i}{2 \beta}\left(\hat{P}_{x x} H_{x}+\hat{P}_{x y} H_{y}\right), \\
& \left(1+\frac{i}{2 \beta} \frac{\partial \ln n^{2}}{\partial z}-\frac{i}{2 \beta} \frac{\partial}{\partial z}\right) \frac{\partial H_{y}}{\partial z}=\frac{i}{2 \beta}\left(\hat{P}_{y x} H_{x}+\hat{P}_{y y} H_{y}\right),
\end{aligned}
$$

where

$$
\begin{gathered}
\hat{P}_{x x} H_{x}=\nabla_{\perp}^{2} H_{x}+\left(k^{2} n^{2}-\beta^{2}-i \beta \frac{\partial \ln n^{2}}{\partial z}\right) H_{x}-\frac{\partial \ln \left(n^{2}\right)}{\partial y} \frac{\partial H_{x}}{\partial y}, \\
\hat{P}_{x y} H_{y}=\frac{\partial \ln \left(n^{2}\right)}{\partial y} \frac{\partial H_{y}}{\partial x} \\
\hat{P}_{y y} H_{y}=\nabla_{\perp}^{2} H_{y}+\left(k^{2} n^{2}-\beta^{2}-i \beta \frac{\partial \ln n^{2}}{\partial z}\right) H_{y}-\frac{\partial \ln \left(n^{2}\right)}{\partial x} \frac{\partial H_{y}}{\partial x} \\
\hat{P}_{y x} H_{y}=\frac{\partial \ln \left(n^{2}\right)}{\partial x} \frac{\partial H_{x}}{\partial y}
\end{gathered}
$$

and $\nabla_{\perp}^{2}=\partial^{2} / \partial^{2} x+\partial^{2} / \partial^{2} y$. In general, the refractive index $n(x, y, z)$ is complex. Equations (6) and (7) contain the second order derivative $\partial^{2} / \partial z^{2}$. This derivative is necessary for the correct description of the phase delay of the fibre modes, whose propagation constants can differ from the reference propagation constant $\beta$.

The Fourier-based BPM (Wangüemert-Pérez et al., 2004) was adapted to take into account the second order derivative $\partial^{2} / \partial z^{2}$ and the variable transverse profile of the PBF. Here we consider the $H_{x}$ component of the magnetic field. The equation 
for $H_{y}$ can be derived in a similar manner. Using $\partial H_{x} / \partial z \simeq\left(H_{x}^{(2)}-H_{x}^{(1)}\right)(\Delta z)^{-1}$, a finite-difference Crank-Nicolson scheme (Thomas, 1995) for Eqn. (6) can be obtained as:

$$
\left(1+\frac{i}{2 \beta} \frac{\partial \ln n^{2}}{\partial z}-\frac{i}{2 \beta} \frac{\partial}{\partial z}\right) \frac{H_{x}^{(2)}-H_{x}^{(1)}}{\Delta z}=i \frac{1}{4 \beta}\left(\hat{P}_{x x} H_{x}^{(2)}+\hat{P}_{x y} H_{y}^{(2)}\right)-i \frac{1}{4 \beta}\left(\hat{P}_{x x} H_{x}^{(1)}+\hat{P}_{x y} H_{y}^{(1)}\right)
$$

where $H_{x}^{(2)}=H_{x}(x, y, z+\Delta z), H_{x}^{(1)}=H_{x}(x, y, z)$. To take into account the effect of the $i(2 \beta)^{-1}(\partial / \partial z)$ term on the beam propagation, the (1,1) Padé approximation (Hadley, 1992) for Eqn. 12 was used. Under the assumption $\left|(2 \beta)^{-2}\left(\partial \ln n^{2} / \partial z\right)^{2}\right| \ll$ 1 , the following equation can be written for $H_{x}$ :

$$
\begin{aligned}
& \left(1+\frac{i}{\beta} \frac{\partial \ln n^{2}}{\partial z}\right) H_{x}^{(2)}+\left(\frac{1}{(2 \beta)^{2}}-i \frac{\Delta z}{4 \beta}\right)\left(\hat{P}_{x x} H_{x}^{(2)}+\hat{P}_{x y} H_{y}^{(2)}\right)= \\
& \left(1+\frac{i}{\beta} \frac{\partial \ln n^{2}}{\partial z}\right) H_{x}^{(1)}+\left(\frac{1}{(2 \beta)^{2}}+i \frac{\Delta z}{4 \beta}\right)\left(\hat{P}_{x x} H_{x}^{(1)}+\hat{P}_{x y} H_{y}^{(1)}\right)
\end{aligned}
$$

The magnetic field $\left(H_{x}, H_{y}\right)$ is expanded into a two-dimensional Fourier series:

$$
H_{x, y}(x, y, z)=\sum_{m, l=-M}^{M} h_{x, y}^{(m, l)}(z) \exp \left(i m \frac{2 \pi}{s} x+i l \frac{2 \pi}{s} y\right)
$$

where $h_{x}^{(m, l)}(z)$ and $h_{y}^{(m, l)}(z)$ are Fourier series coefficients, $M$ is the number of harmonics in each direction, and $s$ defines the rectangular computational window $-s / 2 \leq(x, y) \leq s / 2$, where $s=59.14 \mu \mathrm{m}$ (Fig. 1a). The refractive index profile $n^{2}(x, y, z)$ is expanded into a Fourier series as:

$$
n^{2}(x, y, z)=\sum_{m, l=-2 M}^{2 M} \varepsilon_{m, l} \exp \left(i m \frac{2 \pi \sigma}{s}(x+\xi)+i l \frac{2 \pi \sigma}{s} y\right),
$$

where the parameters $\sigma=\sigma(z)$ and $\xi=\xi(z)$ describe the variation of the core size and the structure shift, respectively. The Fourier series coefficients for $n^{2}(x, y, z)$ and $\ln \left(n^{2}\right)$ are calculated for $\sigma=1$ and $\xi=1$ using the bitmap images of the transverse distribution of refractive index (Fig. 1a). With the change of the factors $\sigma(z)$ or $\xi(z)$, the Fourier coefficients $\varepsilon_{m, l}$ in Eqn. 15 are rescaled analytically. The substitution of (14) and (15) into the finite-difference equations for $H_{x}(13)$ and $H_{y}$ gives origin to sets of linear equations for the unknown Fourier coefficients $h_{x}^{m, n}(z+\Delta z)$ and $h_{y}^{m, n}(z+\Delta z)$, which are computed at each propagation step. A region of absorbing material was placed at the boundaries of the computational window by the addition of a large imaginary part to the index of refraction (Scarmozzino and Osgood, 1991). The absorbing boundary was defined using the Eqn. (4). The initial (launch) beam is a Gaussian:

$$
H_{x}(x, y)_{z=0}=\exp \left(-\left(x^{2}+y^{2}\right) w_{0}^{-2}\right), \quad H_{y}=0,
$$

where the beam radius is $w_{0}=8 \mu \mathrm{m}$.

The refractive index profile of the PBF is a step function. Step discontinuities arise at the boundaries of the rods. At these discontinuities the derivatives $\partial \ln \left(n^{2}\right) / \partial x$ and $\partial \ln \left(n^{2}\right) / \partial y$ in (8-11) give Dirac delta function. Using Fourier series (15) we approximate both the refractive index steps and derivatives $\partial \ln \left(n^{2}\right) / \partial x, \partial \ln \left(n^{2}\right) / \partial y$. The accuracy of the approximation is dependent how large number of harmonics $M$ is used in calculations. To test the accuracy of the beam propagation method the analytical solution for the modes of step-index circular fibre (Snyder et al, 1983) can be used as initial condition. The photonic bands in PBFs arise from the coupled modes of the individual high-index rods (Lægsgaard, 2004). So the numerical method should describe correctly the modes of the rods in the all range of considered wavelengths. With $M=42$ the beam propagation method gives correct phase delay and intensity distribution up to $H E_{31}$ mode of high-index rods which form the considered PBF.

\subsection{Beam Propagation in the PBF}

In this subsection we focus on the modelling of the transverse field distribution in the experimentally realised PBF. The actual PBF structure (Fig. 1a) is considered and the variations of the refractive index profile are neglected, i.e. we assume $\partial \ln n^{2} / \partial z=$ 
0 in Eqns. (6), (7), (8) and (10) and $\sigma(z)=\xi(z)=1$ in Eqn. (15). The near-field outputs obtained after the field propagation in a $60 \mathrm{~mm}$ long section of the PBF are shown in Fig. 7. Figs. 7a and 7d show the transverse intensity distribution at wavelengths located within the transmission bands (Fig. 6). The transverse structure of the field is formed by the PBF mode propagating in a central low index area and by the field guided by high index rods which are acting as step-index fibres. At the wavelength $\lambda=0.59 \mu \mathrm{m}$ (Fig. 7a) the PBF fundamental mode coexists with the modes propagating in six high refractive index rods which surround the PBF core. We found that with the excitation of the fundamental defect mode the polarization of the central area remains linear, while the field inside the high-index rods is polarized elliptically.
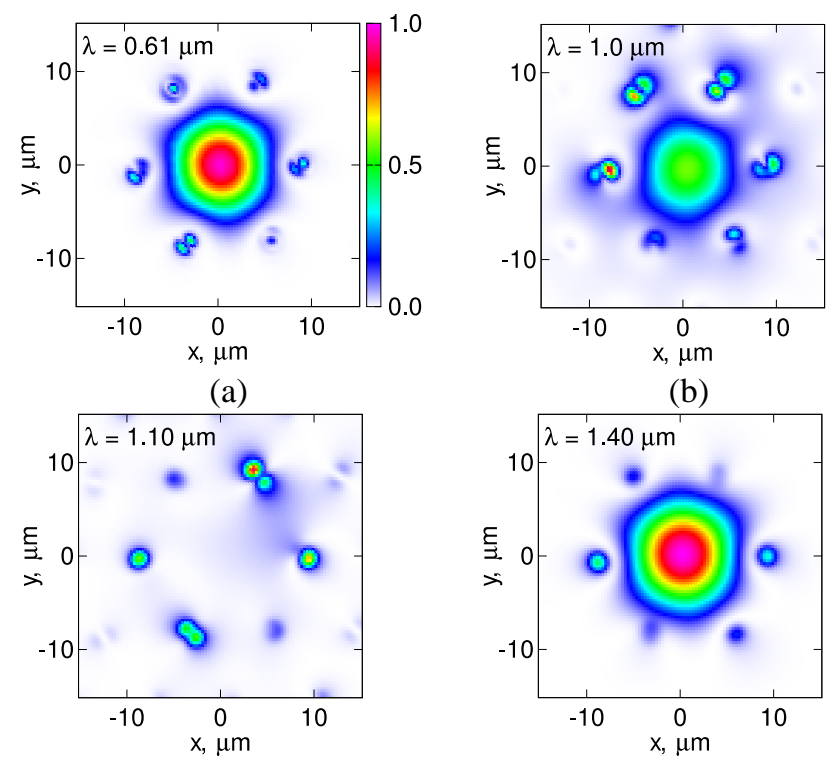

(c)

(d)

Fig. 7 Near-field outputs after a propagation distance $z=60 \mathrm{~mm}$. The transverse intensity distributions are shown for four different operating wavelengths $\lambda$. The color bar in the Fig. 7a shows the intensity color mapping.

At the bandgap edge (Fig. 7b) the field propagates both in the fibre core and in the high refractive index rods surrounding the PBF core. The field guided by the high refractive index rods corresponds to the interference between the $T E_{01}, T M_{01}$ and $H E_{21}$ modes. Out of the bandgap the field is guided by the high refractive index rods (Fig. 7c). The field polarization in the transverse plane is inhomogeneous due to the rod-to-rod deviation of the transverse cross-section in the actual fibre. We found strong field confinement for $\lambda \geq 1.23 \mu \mathrm{m}$. This is in good agreement with the mode calculations reported in Section 2. For this wavelength range the field propagates in the central region and partially within the six high refractive index rods (Fig. 7d).

The beam propagation method shows that part of the field can be partially trapped by high refractive index rods (Figs. 7a, 7b, 7d). To consider the effect of the coexistence of the defect mode and the index guiding modes on the PBF transmittance, the power loss coefficient (3) was calculated and is shown in Fig. 8.

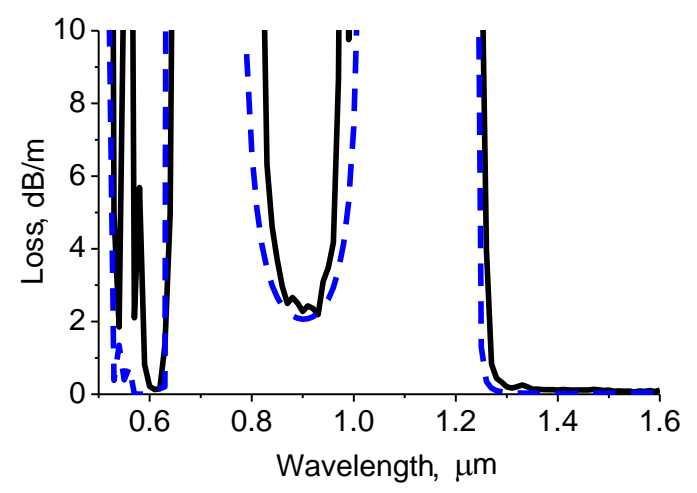

Fig. 8 PBF loss. The solid curve shows the losses calculated using the beam propagation method, while the dashed curve (blue online) reports the losses calculated for the fundamental defect mode using the mode solver (Section 2.2). 
Fig. 8 shows that both the mode solver method (Section 2.2) and the beam propagation method give the same minimum loss values for a given transmission band. Differences exist at the band edges. The excitation of the satellite index guiding modes leads to a narrowing of the transmission bands. At the $3 \mathrm{~dB} / \mathrm{m}$ level the edge of the first transmission band becomes shifted from $1.264 \mu \mathrm{m}$ to $1.249 \mu \mathrm{m}$. The width of the second transmission band calculated using the mode solver is $0.13 \mu \mathrm{m}$, whilst that calculated using the BPM is $0.08 \mu \mathrm{m}$. The width of the third transmission band calculated using the BPM is reduced by a factor of two over that calculated using the mode solver.

\subsection{Effect of the longitudinal variations of the PBF structure on the power transmission}

Here the possible effect of the longitudinal deformations on the field attenuation is considered. In the Fourier series expansion of Eqn. (15) the deformation of the PBF structure is described by the two parameters $\sigma(z)$ and $\xi(z)$. The sine-wave modulation of the core size is given by:

$$
\sigma(z)=1+0.02 \sin \left(2 \pi z / z_{m}\right), \quad \xi(z)=1
$$

where $z_{\mathrm{m}}=400 \mu \mathrm{m}$ is the modulation period. The periodic shift of the whole structure is given by:

$$
\sigma(z)=1, \quad \xi(z)=0.3 \sin \left(2 \pi z / z_{m}\right)
$$

Equations (17) and (18) describe the submicron variations of the PBF structure. We consider these variations of the PBF structure only as one possible scenario for the formation of the large transmission losses. In real fibres the change of the transverse profile can be imposed by drawing conditions, defects in the high-index rods (Schmidt et al., 2009) or air bubbles at the interface between the high-index rods and the host glass, as was observed for a chalcogenide photonic crystal fibre preform by (Brilland et al., 2008). Within the transmission band, the variation of the PBF structure does not noticeably affect the shape of the transverse field distribution, which remains as the one shown in Fig. 7. To consider the effect of the fibre deformation on the fibre transmittance, the fractional power $P_{A} / P_{0}$ was calculated as a function of the propagation distance $z$, where the power of the field propagating in the central area of the PBF is $P_{A}(z)=\int_{0}^{2 \pi} d \varphi \int_{0}^{a} S_{z}(z) r d r, a=4 \mu m, S_{z}(z)=(1 / 2) \operatorname{Re}\left[\boldsymbol{E} \times \boldsymbol{H}^{*}\right]_{z}$ is $z-$ component of the time-averaged Poynting vector, where $\boldsymbol{E}$ and $\boldsymbol{H}$ are the complex amplitudes of electric and magnetic field vector correspondingly. The initial beam power is $P_{0}=\int_{0}^{2 \pi} d \varphi \int_{0}^{\infty} S_{z}(0) r d r$. The results are shown in Fig. 9 for three operating wavelengths; panel (a) refers to a sine-wave modulation described by Eqn. (17), while panel (b) refers to a periodic shift described by Eqn. (18).

Fig. 8 shows that without deformations $(\sigma=\xi=1)$ the confinement losses at the centre of the bandgap regions do not exceed $3 \mathrm{~dB} / \mathrm{m}$. Fig. 9 shows that the variation of the PBF structure, however, leads to an attenuation above $40 \mathrm{~dB} / \mathrm{m}$ for the wavelengths corresponding to the centre of the first $(\lambda=1.6 \mu \mathrm{m})$ and second $(\lambda=0.9 \mu \mathrm{m})$ bandgaps. For the third bandgap $(\lambda=0.61 \mu \mathrm{m})$ the attenuation exceeds $80 \mathrm{~dB} / \mathrm{m}$ for the sine-wave modulation of the PBF structure (Fig. 9a) and $200 \mathrm{~dB} / \mathrm{m}$ for the periodic shift of the whole structure of the PBF (Fig. 9b). This result suggests that the origin of the high losses observed experimentally should be attributed primarily to defects in the manufactured fibre rather than to a confinement attenuation or an intrinsic material absorption.

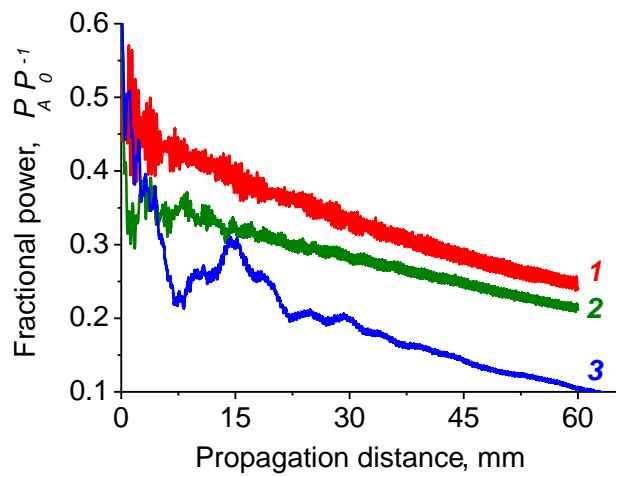

(a)

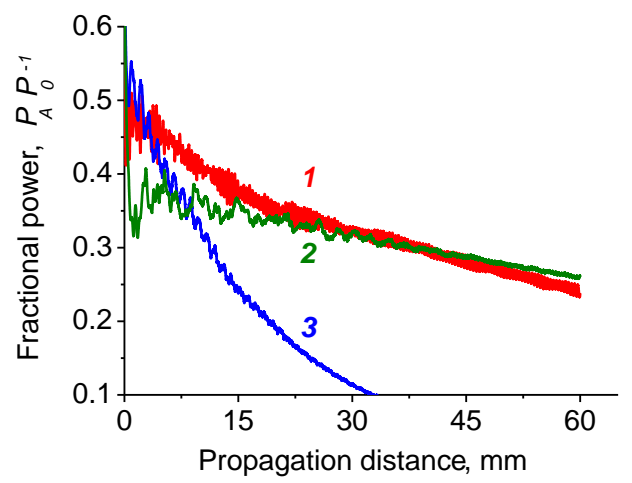

(b)

Fig. 9 The fractional power of the field propagating in the central area of the PBF. The curve 1 (red online) corresponds to the wavelength $\lambda=1.6 \mu \mathrm{m}$, curve 2 (green online) is for $\lambda=0.9 \mu \mathrm{m}$, curve 3 (blue online) is for $\lambda=0.61 \mu \mathrm{m}$. (a) Power depletion under a sine-wave modulation of the PBF structure described by Eqn. (17); (b) Power depletion under a periodical shift of the whole structure, as described by Eqn. (18). 


\section{Conclusion}

We have analysed the transmittance for a real PBF refractive index profile and compared it with the transmittance of an idealized hexagonal structure. The submicron structural distortions of the fibre cross-section significantly impact only on the transmission bandwidth; the confinement loss does not change significantly within the selected bandgap. Our analysis shows that submicron structure variations along the fibre length are more significant than the submicron deformations of the transverse PBF profile. Longitudinal variations of the fibre structure can increase transmission losses by up to tens of $\mathrm{dB} / \mathrm{m}$. We found that at the bandgap edges the fundamental defect mode coexists with the index-guiding modes of the rods that surround the PBF core (and which act as step-index fibres). The excitation of the index-guiding modes narrows the transmission bandwidth. The effect of the narrowing of the transmission band is minimal for the first-order bandgap but increases with the bandgap index. We found that low-loss guidance within the bandgap wavelengths can be obtained even with the deformed PBF cross-section.

Acknowledgements A. Konyukhov is grateful for the grant of the Directorate of the Program of International Training Fellowships of the Saratov State University, supported by the Ministry of Education and Science, Russian Federation.

\section{References}

Argyros, A., Birks, T.A., Leon-Saval, S.G., Cordeiro, C.M.B., Luan, F., Russell, P.St.J.: Photonic bandgap with an index step of one percent. Opt. Exp. 13, 309-314 (2005)

Birks, T.A., Luan, F., Pearce, G.J., Wang, A., Knight, J.C., Bird, D.M.: Bend loss in all-solid bandgap fibres. Opt. Exp. 14, 5688-5698 (2006)

Brilland, L., Troles, J., Houizot, P., Désévédavy, F., Coulombier, Q., Renversez, G., Chartier, T., Nguyen, T.N., Adam, J., Traynor, N.: Interfaces impact on the transmission of chalcogenides photonic crystal fibres. J. of the Ceram. Soc. of Jap. 116, 1024-1027 (2008)

Caillaud, C., Renversez, G., Brilland, L., Mechin, D., Calvez, L., Adam, J.-L., Troles, J.: Photonic bandgap propagation in all-solid chalcogenide microstructured optical fibers. Materials 7, 6120-6129 (2014).

de Oliveira, R.E.P., Knight, J.C., Taru, T., de Matos, C.J.S.: Temperature response of an all-solid photonic bandgap fiber for sensing applications. Appl. Opt. 52, 1461-1467 (2013)

Eliseev, M.V., Rozhnev, A.G., Manenkov, A.B.: Guided and leaky modes of complex waveguide structures. J. Lightw. Technol. 23, 2586-2594 (2005)

Guobin, R., Zhi, W., Shuqin, L., Yan, L., Shuisheng, J.: Full-vectorial analysis of complex refractive-index photonic crystal fibers. Opt. Expr. 12, 1126-1135 (2004)

Hadley, G.R.: Wide-angle beam propagation using Padé approximant operators. Opt. Lett. 17, 1426-1428 (1992)

Hill, C., Jha, A.: Development of novel ternary tellurite glasses for high temperature fiber optic mid-IR chemical sensing. J. of Non-Crystalline Solids 353, 1372-1376 (2007)

Lehoucq, R.B., Sorensen, D.C., Yang, C.: ARPACK Users' Guide: Solution of Large Scale Eigenvalue Problems with Implicitly Restarted Arnoldi Methods. Houston, Rice Univ. (1997) Available:

http://www.caam.rice.edu/software/ARPACK/UG/ug.html

Li, M.-J., West J.A., Koch, K.W.: Modeling effects of structural distortions on air-core photonic bandgap fibers. J. Lightw. Technol. 25, 2463-2467 (2007)

Lousteau, J., Scarpignato, G., Athanasiou, G.S., Mura, E., Boetti, N., Olivero, M., Benson, T., Sewell, P., Abrate, S., Milanese, D.: Photonic bandgap confinement in an all-solid tellurite-glass photonic crystal fibre. Opt. Lett. 37, 4922-4924 (2012)

Lægsgaard, J.: Gap formation and guided modes in photonic bandgap fibres with high-index rods. J. Opt. A: Pure Appl. Opt. 6 798-804 (2004) 
Manenkov, A.B.: Orthogonality conditions for leaky modes. Radiophys. and Quant. Electron. 48 348-360 (2005)

Melnikov, L., Khromova, I., Scherbakov, A., Nikishin, N.: Soft-glass hollow-core photonic crystal fibres. Proc. SPIE 5950, $595012(2005)$

Mouawad, O., Amrani, F., Kibler, B., Picot-Clémente, J., Strutynski, C., Fatome, J., Désévédavy, F., Gadret, G., Jules, J-C., Heintz, O., Lesniewska, E., Smektala, F.: Impact of optical and structural aging in $\mathrm{As}_{2} \mathrm{~S}_{3}$ microstructured optical fibers on mid-infrared supercontinuum generation. Opt. Exp. 22, 23912-23919 (2014)

Pureur, V., Bouwmans, G., Perrin, M., Quiquempois, Y., Douay, M.: Impact of transversal defects on confinement loss of an all-solid 2-D photonic-bandgap fiber. J. Lightw. Technol. 25, 3589-3596 (2007)

Ren, G., Shum, P., Zhang, L., Yu, X., Tong, W., Luo, J.: Low-loss all-solid photonic bandgap fiber. Opt. Lett. 32, 1023-1025 (2007)

Sakoda, K.: Optical Properties of Photonic Crystals. Springer, Berlin (2001)

Saitoh, S., Saitoh, K., Kashiwagi, M., Matsuo, S., Dong, L.: Design optimization of large-mode-area all-solid photonic bandgap fibers for high-power laser applications. J. Lightw. Technol. 32, 440-449 (2014)

Scarmozzino, R., Osgood, R.M.: Comparison of finite-difference and Fourier-transform solutions of the parabolic wave equation with emphasis on integrated-optics applications. J. Opt. Soc. Am. A 8, 724-731 (1991)

Schmidt, M.A., Granzow, N., Da, N., Peng, M., Wondraczek, L., Russell, P.St.J.: All-solid bandgap guiding in telluritefilled silica photonic crystal fibers. Opt. Lett. 34, 1946-1948 (2009)

Snopatin, G.E., Churbanov, M.F., Pushkin, A.A., Gerasimenko, V.V., Dianov, E.M., Plotnichenko, V.G.: High purity arsenic-sulfide glasses and fibers with minimum attenuation of 12 dB/km. J. Opt. Adv. Mater. Rapid Commun. 3, 669-671 (2009)

Snyder, A.W., Love J.D.: Optical waveguide theory. Chapman and Hall, London (1983).

Thomas, J.W.: Numerical Partial Differential Equations: Finite Difference Methods. Texts in Applied Mathematics. 22, Berlin, New York: Springer-Verlag (1995)

Toupin, P., Brilland, L., Méchin, D., Adam, J.-L., Troles, J.: Optical aging of chalcogenide microstructured optical fibers. J. Lightw. Technol. 32, 2428-2432 (2014)

Xue, S.C., Large, M.C.J., Barton, G.W., Tanner, R.I., Poladian, L., Lwin, R.: Role of material properties and drawing conditions in the fabrication of microstructured optical fibers. J. Lightw. Technol. 24, 853-860 (2006)

Vanvincq, O., Kudlinski, A., Bétourné, A., Mussot, A., Quiquempois, Y., Bouwmans, G.: Manipulating the propagation of solitons with solid-core photonic bandgap fibers. Int. Journal of Optics 2012, 157319 (2012)

Wangüemert-Pérez, J.G., Molina-Fernández, I., Luque-Nieto, M.A.: A novel Fourier based 3D full-vectorial beam propagation method. Opt. and Quant. Electron. 36, 285-301 (2004)

Yan, P.-G., Zhao, J., Ruan, S.-C., Yu, Y.-Q., Shu, J., Wu, X., Ding, J.F., Wei, H.-F., Luo, J.: Drawing an ultra-low loss allsolid photonic bangap fiber for ytterbium ASE suppression. Microw. Opt. Technol. Lett. 52, 2629-2632 (2010) 